\title{
A Novel Method using Long Axis Cardiac MRI Measurements can Improve in vivo Myocardial Infarct Quantification
}

Jacob Ref ( $\square$ jref13@email.arizona.edu )

The University of Arizona College of Medicine Tucson https://orcid.org/0000-0002-7381-2099

\section{Sherry Daugherty}

University of Arizona Medical Center - University Campus: Banner University Medical Center Tuscon Ikeotunye Royal Chinyere

The University of Arizona College of Medicine Tucson

Janan Zeng

The University of Arizona College of Science

Jordan J Lancaster

University of Arizona Medical Center - University Campus: Banner University Medical Center Tuscon

\section{Laryenth Lancaster}

UMC: Banner University Medical Center Tuscon

Kenneth Fox

UMC: Banner University Medical Center Tuscon

\section{Mark Borgstrom}

The University of Arizona

\section{Adrian Grijalva}

University of Arizona Health Sciences Library: The University of Arizona Health Sciences

\section{Jen Koevary}

University of Arizona Medical Center - University Campus: Banner University Medical Center Tuscon

\section{Steven Goldman}

University of Arizona Medical Center - University Campus: Banner University Medical Center Tuscon

Ryan Avery

Northwestern University Feinberg School of Medicine

\section{Research Article}

Keywords: AHA 17-Sgement Model, Ischemic Heart Failure, MRI, Long-Axis Ratio, Histopathology, Myocardial Infarction

Posted Date: September 21st, 2021 
DOI: https://doi.org/10.21203/rs.3.rs-899871/v1

License: (c) (1) This work is licensed under a Creative Commons Attribution 4.0 International License. Read Full License 
Title:

A Novel Method using Long Axis Cardiac MRI Measurements can Improve in vivo Myocardial Infarct Quantification

Brief Title:

LAX MRI Infarct Sizing

Manuscript Word Count: 2,851

Authors \& Affiliations:

Jacob Ref a, BS, Sherry Daugherty c ${ }^{\mathrm{c}}$, BS, Ikeotunye Royal Chinyere ${ }^{\mathrm{d}}$, PhD, Janan Zeng ${ }^{\mathrm{i}}$, Jordan J Lancaster $^{\mathrm{c}}$, PhD, L.D. Lancaster ${ }^{\mathrm{g}}$, MD, Kenneth Fox ${ }^{\mathrm{g}}$, MD, Mark Borgstrom ${ }^{\mathrm{h}}, \mathrm{PhD}$, Adran Grijalva $^{\mathrm{i}}$, BS, Jen Watson Koevary ${ }^{\mathrm{c}}$, PhD, Steven Goldman ${ }^{\mathrm{f}}$, MD, Ryan Avery ${ }^{\mathrm{b}}$, MD

a. MD Program, College of Medicine, University of Arizona, Tucson, AZ

b. Feinberg School of Medicine, Northwestern University, Chicago, IL

c. Sarver Heart Center, University of Arizona, Tucson, AZ

d. MD-PhD Program, College of Medicine, University of Arizona, Tucson, AZ

e. Department of Biomedical Engineering, University of Arizona, Tucson, AZ

f. Division of Cardiology, Banner - University Medical Center, Tucson, AZ

g. Division of Cardiothoracic Surgery, Banner - University Medical Center, Tucson, AZ

h. Information Technology Services, University of Arizona, Tucson, AZ

i. Department of Physiology, University of Arizona, Tucson, AZ

* No conflicts of interest nor disclosures exist for all authors 
Corresponding Author:

Jacob Ref

Sarver Heart Center, University of Arizona

1501 North Campbell Avenue, Room 6154

Tucson, AZ, 85724

P: (602)-576-5585

jref13@email.arizona.ed 


\begin{abstract}
:
Purpose

Currently, the American Heart Association (AHA) 17-segment model is the preferred clinical method to define and quantify left ventricle (LV) myocardial infarction (MI) size. This method is subjective and can be inaccurate given that segmental approximation assumes a specific percent of infarcted tissue when compared to reference standard post-mortem histopathology.
\end{abstract}

To improve the accuracy and reproducibility of infarct volume quantification we propose a novel measurement technique based on cardiac MRI images from a porcine model of myocardial infarction. Data were collected from serial MRI exams of Yucatan mini swine over 6 months and endpoint organ harvesting for histopathologic analysis.

Methods

Two observers evaluated four infarct sizing methods: myocardial contouring of post-mortem heart slices, contouring using cardiac MRI, AHA 17-segment model analysis and novel long-axis MRI infarct sizing.

Results 
LV infarct sizes ranges were $1.6 \%-25.8 \%(n=10)$ using reference standard histopathologic infarct sizing. Intraclass correlations (ICC) were calculated between two observers and averaged due to high similarity, ICC $>.900$. A t-test of .0006 and Bland-Altman plots show statistically significant differences in 17-segment model infarct size compared to histopathologic analysis while no significant difference was found when compared to our new novel method with 0.8198 . Linear correlation showed an $\mathrm{R}^{2}$ of 0.9111 between MRI contoured infarct size and our novel MRI infarct sizing model to predict infarct size as a percentage while the $\mathrm{R}^{2}$ of the $17-\mathrm{Seg}$ model is 0.8197 .

\section{Conclusions}

The 17-sgement model provides an inferior quantitative assessment of LV infarct size compared to the proposed long-axis infarct sizing suggesting it maybe a robust and easily implementable quantitative assessment of LV infarct size in advanced imaging.

Keywords: AHA 17-Sgement Model; Ischemic Heart Failure; MRI; Long-Axis Ratio; Histopathology; Myocardial Infarction 


\section{Declarations}

Funding:

This work was supported by the Arizona Biomedical Research Commission (RFGA No.

ADHS16-00005489), WARMER Research Foundation, and the Sarver Heart Center, University of Arizona.

Conflicts of interest/Competing interests:

There are no conflicts of interest or competing interests for the authors listed on this manuscript.

\section{Availability of data and material:}

All data supporting the conclusions of this study are contained within the manuscript and inquires beyond the manuscript can be made to the first author.

\section{Code availability:}

Not applicable.

\section{Author's Contributions:}

Jacob Ref, BS - first author, data collection and manuscript drafting

Ryan Avery, MD - senior physician scientist, provided scientific guidance

Sherry Daugherty - assisted with manuscript drafting

Jordan J Lancaster, $\mathrm{PhD}$ - research scientist, obtained funding, assisted with manuscript drafting Ikeotunye Royal Chinyere, $\mathrm{PhD}$ - assisted with manuscript drafting and provided clinical context Jen Koevary, PhD - research scientist, obtained funding, assisted with manuscript drafting 
Steven Goldman - senior physician scientist, obtained funding, provided scientific guidance Janan Zeng - assisted with manuscript drafting and data collection Adrian Grijalva, BS - assisted with manuscript drafting Mark Borgstrom, $\mathrm{PhD}$ - research scientist, assisted with statistical analysis Larynth Lancaster, MD - senior physician scientist, provided scientific guidance Kenneth Fox, MD - senior physician scientist, provided scientific guidance

\section{Ethics Approval:}

Research oversight and approval was conducted by Institutional Animal Care and Use Committee. University Animal Care provided veterinary care, husbandry and surgical oversight within their facilities that are accredited and registered by the Association for Assessment and Accreditation of Laboratory Animal Care International (AAALACi Number (accredited since 1969): 000163, Continuing Full Accreditation (effective March 4, 2020)), Public Health Service (NIH/OLAW)(PHS Animal Welfare Assurance Number: D16-00159 (A-3248-01), Effective August 8, 2019, Expires August 31, 2023) and the United States Department of Agriculture (USDA Animal Research Facility Registration Number: 86-R-0003, Expires August 24, 2022).

\section{Consent to Participate:}

Not applicable.

\section{Consent to Publish:}

Not applicable. 
Acknowledgements:

A special thank you to Alice McArthur, Arielle Tran, and Grace Gorman for their technical assistance. 


\section{Introduction}

Late gadolinium enhancement (LGE) imaging of the left ventricular myocardium using long and short axis images obtained with cardiac magnetic resonance imaging (MRI) has increasingly become a part of routine clinical assessment of both ischemic and nonischemic cardiomyopathies [1]. The LGE images have become a rapidly evolving technique for assessment of patterns and extent of myocardial fibrosis, particularly myocardial infarction (MI). This method to noninvasively detect myocardial infarctions has improved both clinical diagnosis of ischemic cardiomyopathy, and the ability to characterize the location of and size of myocardial infarct [2, 3]. While the precision of infarct-sizing continues to advance with emerging imaging techniques $[4,5]$, the clinical standard for communicating MI size and location continues to use the methods proposed by the 17-segment model provided by American Heart Association (AHA) [1]. The 17-segment model has continued to be a readily accessible technique for rapid qualitative analysis of infarct size and location; however, this method has shown shortcoming when attempting to provide definitive quantitative analysis of myocardial infarct size $[6,7,8]$.

The previously described limitations of the 17-segment model have focused overestimation of infarct size because most MIs do not truly conform to a proposed segmental distribution as defined by the 17-segment model $[6,9,10,11,12]$. Standard quantification of LV infarct sizing can be performed by image post-processing that involves contouring both the infarct and left ventricular margins; however, this method is often too tedious and time-consuming to be done routinely in clinical practice $[6,10,11,12]$. 
We propose a novel method utilizing the multiple routine views acquired during LGE sequence acquisitions during standard cardiac MRI. Since the images evaluate the infarct in multiple dimensions, they contain extensive data can be used for infarct size and quantification. This novel method for myocardial infarct quantification uses 4 defined measurements from routine long-axis LGE images to produce a LAX ratio value (LAR). Given the advantages of the proposed myocardial infarct sizing method using LAX images, it is expected to provide an easily implementable method for clinical assessment of ischemic heart disease that could guide both cardiac diagnosis and treatment strategies. Furthermore, this new method is expected to provide a more accurate method for scar quantification and estimation with robust application to both clinical and research methodologies. 


\section{Methods}

\section{Myocardial Infarction and Endpoint Procedures}

This study was performed as a component of an institutionally reviewed and approved trial evaluating ischemic heart failure in a cohort of 13 male Yucatan mini swine over a period of 8 months. Data are taken from a swine model simulating chronic ischemic heart failure performed by a $90 \mathrm{~min}$ balloon occlusion/reperfusion procedure involving the left anterior descending (LAD) coronary artery inducing an anterior LV wall infarct in the LAD vascular territory. Infarct sizes are variable due to multiple factors including balloon placement and collateral network. This procedure was performed with the assistance of a surgical group comprised of a cardiologist, cardiothoracic surgeon, and surgical technicians, and included a coronary artery angiogram to localize the coronary arteries at the time of procedure to ensure proper placement of the catheter-directed balloon that was used to perform the LAD occlusion just distal to the first diagonal. A left ventriculogram was performed after the MI to document hypokinesia of the LV anterior wall.

All Cardiac MRI (CMR) exams were acquired on a 3T Magnetom Skyra (Siemens Healthineers, Erlangen, AG). Each mini swine underwent a series of 5 CMRIs obtained at baseline, 1-month post MI, and 1-month, 3-months, and 6-months post-surveillance. An endpoint necropsy was performed three days following the 6-month post-surveillance MRI in order to obtain histopathology of the infarcted heart for analysis. Three swine were excluded due to insignificant 
LV myocardial scarring found in MRI and histopathology. The included swine all had MI greater than $12 \%$ of total $\mathrm{LV}$ volume $(\mathrm{n}=10)$.

AHA 17 Segment Model

In 2002, the AHA introduced a model for localization of size and regional distribution when estimating left ventricle (LV) infarction. The AHA model utilizes the SAX and LAX images of the heart to divide the left ventricle into 17 distinct segments proceeding from base to apex (Figure 1A) while utilizing the four walls of the left ventricle [1].

By convention, each segment accounts for $5.88 \%$ of the total myocardium of the left ventricle, and the segments can be cumulatively added for an approximation of total infarct percentage if either a portion of or the complete segment of myocardium is infarcted. Sizing is done utilizing LGE sequences on MRI or other advanced imaging to assess ventricular abnormalities, such as echocardiogram or nuclear myocardial perfusion imaging. The 17-segment model is a wellaccepted model to assess location and size of infarcted myocardium although lacks accuracy when quantifying the percentage of infarcted tissue given that the summation of segments can overestimate infract volume since partial segments are seen as being completely affected [7].

Infarct Sizing in Cardiac MRI

Standard volumetric quantification of myocardial tissue volume and infarct volume, utilizes myocardial LGE sequence to provide measurements of the epicardium, endocardium and infarct 
utilizing the amount of myocardial LGE as it relates to normal appearing myocardium in a stack of CMR short-axis (SAX) LGE images that cover the entire length of the left ventricle (figure 1B). The SAX LGE MRI images allow for comprehensive evaluation of the entire LV by extending from the LV outflow track to an area just distal to the true apex of the LV. This LV coverage is a conventional method that includes a stack of 8-12 MRI images of 0.4-centimeter thickness. QuPath (Edinburgh, Scotland) was used as a standard processor for manual tracing of LV segments.

In this approach, for every slice of the LGE image stack the epicardium of the LV is manually contoured resulting in a total area of the LV myocardium and cavity, which is denoted by the green outline in Figure 1B. The endocardium is also manually traced for each image resulting in the total area the LV cavity, denoted by the red outline in Figure 1B. Subsequently, the area defined by the green line (the LV myocardium and cavity) is subtracted by the area defined by the red line (the LV cavity) to yield the area of LV myocardium. The margins of LGE, denoting the site fibrosis related to MI, are then traced on each image of the SAX stack, which is denoted by the yellow outlined area in Figure 1B.

Measurements of MI area and LV myocardium area were summated to be representative of total volume of LV infarct as it related to a total volume of LV myocardium. Finally, the total area of MI was divided by total LV myocardium to yield an LV infarct percentage.

$$
L V \text { Infarct Percentage }(M R I)=\frac{\text { Total Myocardial Infarction Area }\left(\text { Yellow }^{\wedge}\right)}{\text { Total LV Myocardium Area }\left(\text { Green }^{\wedge}-\text { Red }^{\wedge}\right)} * 100
$$




\section{$\wedge=$ reference colors from Figure 1B}

\section{Infarct Sizing in Post-Mortem Heart Slices}

In order to compare what endpoint MRI data acquired by the volumetric analysis of the SAX LGE images to terminal histopathology, the following post-mortem heart analysis was done [13]. The pathologic specimen of the swine heart was acquired in toto 3 days following the endpoint MRI performed on surveillance MRI performed 6 months after infarction. The heart was sliced into 4 discrete contiguous segments, with each segment approximately 0.5 -centimeters in thickness, to simulate the standard thickness of the SAX MRI stack images.

Measurements were performed using the apical and basal sides of the 4 slices to increase correlation with CMR images. QuPath (Edinburgh, Scotland) was used as a standard processor for manual tracing of LV segments.

In a similar fashion to the method infarct sizing on the LGE SAX MRI sequences, the epicardium of the LV was contoured for each image resulting in a total area of the LV myocardium and cavity, which is denoted by the green outline in Figure 1C. The endocardium was then manually traced for each image resulting in a total area the LV cavity, denoted by the red outline in Figure 1C. Subsequently, the area defined by the green line (the LV myocardium and cavity) was subtracted by the area defined by the red line (the LV cavity) to yield the area of LV myocardium. The area of MI was then traced, denoted by the yellow outlined area in Figure $1 \mathrm{C}$. 
Measurements in post-mortem heart slices were performed in a slice limited to the atrioventricular groove to the ventricular apex in order to best match measurements of the CMR evaluation of the left ventricle that extended from the LV outflow track to the true apex. Measurements of MI area and LV myocardium area were summated to be representative of total LV infarct. Finally, the total area of MI is divided by total LV myocardium to yield an LV infarct percentage.

$$
\begin{gathered}
\text { LV Infarct Percentage }(\text { Histology })=\frac{\text { Total Myocardial Infarction Area }\left(\text { Yellow }^{\wedge}\right)}{\text { Total LV Myocardium Area }\left(\text { Green }^{\wedge}-\text { Red }^{\wedge}\right)} * 100 \\
\wedge=\text { reference colors from Figure } 1 \mathrm{C}
\end{gathered}
$$

Long Axis MRI Sizing with Calculation of Long Axis Ratio

LGE images of the LAX were routinely acquired during each MRI to better delineate the infarct, when compared to orthogonal SAX LGE images. The LAX MRI image can be utilized to measure the length of the induced LV infarct along each wall of the LV. Routine 2-, 3- and 4Chamber LAX LGE were acquired with each image oriented to the LV in a previously predefined anatomic plane. These three planes include the anterior and inferior walls (Figure 2A), anteroseptal and inferolateral walls (Figure 2B), anterolateral and inferolateral walls (Figure 2C) of the heart. From each plane, the actual length of the myocardial infarct could be exactly measured by differentiating the area of hyper enhancing infarct that contrasts with normal 
myocardium. This helps to provide exact anatomic location of MI along each wall of the heart relative to the apex.

Total heart length (THL) from apex to base is measured in the 2-Chamber LAX MRI (Figure 2D) in order to standardize processing and provide a more accurate LV infarct percentage, since not every heart is the exact same size and infarct percentage is calculated relative to specific anatomy.

The infarct length measurements from each of the 3 LAX planes are subsequently summated and then divided by THL to produce a Long-Axis Ratio (LAR) value. LAR values were plotted against MRI contoured infarct size in figure 3 to produce a conversion table, table 1, to produce an LV infarct percentage using the calculated LAR value.

$$
\text { LAR Value }=\frac{\begin{array}{c}
2 \text { Chamber Infarct Length }+3 \text { Chamber Infarct Length } \\
+4 \text { Chamber Infarct Length }
\end{array}}{\text { Total Heart Length }(T H L)}
$$

LAX processing from 3 planes gives the observer length of myocardial infarct along each wall and provides an estimated infarct percentage using table 1.

\section{Statistical Analysis}

Two observers processed all infarct-sizing data and intraclass correlations were calculated. High intraclass correlations allowed the data to be averaged in Table 2. Student's $t$-tests were done on 
data to show significant differences in measuring techniques since they were expected to give similar values and no statistical difference is expected.

Bland-Altman plots, in Figure 4, were constructed for both AHA 17-segment model infarct size compared to histopathology infarct size acquired from post-mortem heart slices and for MRI contoured infarct size compared to histopathology infarct size with limits of agreement. Histopathology data were considered the gold-standard for the infarct percentages calculated and is compared to the two measurement techniques. Bland-Altman plots were expected to show small limits of agreement to show reliability of the measuring technique and have data points remain close to zero to demonstrate validity in the measuring technique.

Table 1 has been constructed using the linear trendline derived from Figure 3A to provide a conversion of LAR values to LV infarct percentage, a novel way to calculate LV infarct percentage. 


\section{Results:}

Data from Table 2 shows average infarct sizing done from histopathology contouring, MRI contouring and 17 segment data. Values were averaged between two observers because high intraclass correlations were present. The intraclass correlation were as follows: histopathology infarct sizing: 0.939, 17-Segment model terminal MRI infarct sizing: 0.993, MRI contour terminal MRI infarct sizing: 0.996.

Newly established infarct LAR values had an intraclass correlation of 0.991 between two observers to provide the data. LAR values averaged a value of $1.4 \pm 0.08$ and ranged from $0.0-$ 2.5 and MRI contoured infarct percentages averaged $12.0 \pm 0.8 \%$ and ranged from $0.6-25.3 \%$. LAR values were plotted against MRI contoured infarct percentages in Figure 3A as a scatter plot with both these measurements having been taken in terminal MRIs. The $\mathrm{R}^{2}$ value was very high (0.911). A linear regression was performed plotting the LAR values against MRI contoured infarct percentages to predict LV infarct percentage using LAR values (Table 1). This table is the basis to quantify LV infarct percentages using LAX MRI.

17-segment model infarct sizes values were plotted histopathology infarct sizes and the linear regression yielded an $\mathrm{R}^{2}$ of 0.8197 .

Student's paired t-test values were calculated to report any significant differences between data in Table 2. No significant differences were expected since all infarct sizing techniques were done on the same cases. 
A significant difference $(\mathrm{p}$ value $=<0.05)$ between histopathology infarct sizing and 17Segment model terminal MRI infarct sizing was found.

Bland Altman plots, demonstrated in Figure 4, found evidence supporting the significant difference of the 17 -segment model with wide limits of agreement and a mean ranging from 0 at around 12, (Figure 4A) suggesting the17-segment model measures infarct percentage quite differently compared to reference standard histopathology infarct size measurements, suggesting low reliability in its limits of agreement and low validity with a Bland Altman mean of around 12. The limits of agreement are smaller for and MRI contoured infarct size versus histopathology infarct size with a mean much closer to 0, shown in Figure 4B. MRI contoured infarct size values showed both validity and reliability and were plotted against LAR values in the scatter plot of Figure 3A.

An MRI on a patient with an anterior wall infarct shows that the LAR method has clinical relevance and could be used in patients (Figure 5). 


\section{Discussion:}

The AHA 17-segment model has become a well-validated tool to clinically assess the size of left ventricular myocardial infarction while providing a semi-quantitative estimation of the percent of the total infarcted myocardium [14]. While the model has proven to be useful, it has many accepted limitations regarding the accuracy of quantified estimate of infarct size that warrants further investigation. Given improvements in advanced cardiac imaging techniques, such as cardiac computed tomography and MRI, image postprocessing techniques have been derived that potentially could provide more accurate estimates of ventricle size and function. Furthermore, the use of endocardial and epicardial contouring on MRI has been validated to be the most accurate in-vivo assessment of ventricle size and function providing a new reference standard [15].

Given these advances, utilizing short-axis stacks of LGE imaging provides a similar reference standard for quantification of myocardial infarct volume while providing an unrivaled level of anatomic localization of the infarct [16]. Despite the significant advance provided by LGE quantification of the amount of myocardial hyperenhancement used for myocardial infarct sizing requires dedicated post-processing software and that requires an experienced cardiovascular imager to perform, which currently is often too cumbersome for routine clinical use. The need for time-consuming contouring of ventricular margins along with the myocardial enhancement margins for MI quantifying limits its use to mostly imaging-based research. While advances in artificial intelligence, including deep learning and convolutional neural networks, are expected to 
provide improvements and more efficient tools for image analysis allowing for automated, inline analysis of LGE imaging, current assessment of infarct size and location continues to be performed by the more qualitative approach of the 17-Segment model.

Given the limitations of the 17-segment model, while considering the advances available for detection and localization of infarct provided by cardiac MRI, an improved model for scar quantification that could mirror the easy implementation of the 17-segment model while more accurately evaluating the multiple intrinsic parameters provided by cardiac MRI appears to provide a more accurate assessment of size and volume of myocardial infarction. Presented is a novel model of left ventricular myocardial infarction using the multiple long-axis based LGE images routinely acquired during cardiac MRI viability examination, which can be utilized to estimate the LAR value. Furthermore, our preliminary results suggest this novel method for infarct sizing can provide a more accurate and precise estimate of infarct size and location, thus serving as a promising technique that has several advantages to the 17 -segment model. Infarct size measurements in LAX MRI provide estimations of infarct size along each wall of the heart relative to the apex, providing a more precise infarct locating tool as well.

Our observations are based on data accrued through LGE MRI images that estimate ventricular size and mass utilizing standard quantitative analysis acquired by contouring the left ventricular endocardial and epicardial margins. Infarct quantification was performed in a similar fashion by contouring the exterior margin of myocardial infarct as delineated by areas of hyperenhancement on LGE cardiac MRI images. The collected data were compared to a reference standard of post- 
mortem histopathologic specimens via t-test and Bland-Altman analysis to correlate with ventricular mass and infarct mass. All cardiac MRI examinations in this study were performed within three days prior to autopsy and post-mortem histopathologic analysis; therefore, the analysis of ventricular mass and infarct mass generated by histopathologic analysis was expected to show a strong correlation when compared to conventional MRI contouring methods. The novel LAX infarct sizing method showed strong correlation with histopathologic analysis as well. While estimation of infarct mass with the 17-segment model was expected to be acceptable, it markedly underperformed with a t-test p-value of 0.0006 which was counter to expectations that no statistical difference would be present.

Given our data, infarct sizing MRI utilizing the 3 routine LAX planes acquired during LGE images during cardiac MRI provided a robust, and relatively simple quantitative analysis of MI size as opposed to the cumbersome post-processing reliant quantitative analysis that used the 812 stacked image provided by SAX LGE MRI imaging secondary to the brevity of individual image analysis, the extent of myocardium dimension able to evaluated with LAX views, and the ability to forego extensive post-processing analysis.

When combined with the novel method, this method can provide a standardized infarct percentage calculation across many different patients thus providing both clinicians and researchers a more accurate LV infarct percentage sizing method despite differences in cardiac specimens. While further study is needed, this technique is promising since LAR analysis can be 
done in human subjects, an example is shown in Figure 5, that can estimate anatomic location of MI and provide an estimate of LV infarct percentage.

While this work is promising, limitations were detected. While each swine was done prospectively with histopathological standards, we acknowledge that the low number of swine $(n=10)$ will need to increase in order to further improve the accuracy of the correlation between the novel methods and histologic scoring. All swine provided in this study underwent only LAD occlusion/reperfusion and all infarctions were localized to same vascular territory of the heart. Furthermore, the technique made exclusively transmural infarcts. While this would not be expected to significantly change the data related to infarct localization using the provided novel LAX method; however, further study incorporating nontransmural infarction is needed. While MRI was used since LGE images provides the most accurate in vivo analysis of the infarct and would be able to confirm accuracy when correlated to histopathologic specimens, further study evaluating other advanced imaging modalities is also necessary.

\section{Conclusion:}

Overall, the proposed method of assessment and measurements acquired from 3 long-axis planes LGE MRI images is robust and broadly applicable. This technique holds promise to qualitatively define myocardial infarct size and location given that is provides more accurate LV infarct percent estimations compared to current models. 


\section{References:}

[1] Wagner A, Mahrholdt H, Sechtem U, Kim RJ, Judd RM. MR imaging of myocardial perfusion and viability. Magn Reson Imaging Clin N Am. 2003 Feb;11(1):49-66. doi: 10.1016/s1064-9689(02)00048-x. PMID: 12797510.

[2] Piotr J. Slomka, PhD; David Fieno PhD, MD; Louise Thomson, MD; John D. Friedman, MD; Sean W. Hayes, MD; Guido Germano, PhD; Daniel S. Berman, MD. Automatic Detection and Size Quantification of Infarcts by Myocardial Perfusion SPECT: Clinical Validation by Delayed-Enhancement MRIJ Nucl Med 2005 46:728-735

[3] Kim RJ, Wu E, Rafael A, Chen EL, Parker MA, Simonetti O, et al. The use of contrastenhanced magnetic resonance imaging to identify reversible myocardial dysfunction. $\mathrm{N}$ Engl $\mathbf{J}$ Med. 2000;343(20):1445-53.

[4] López-Fernández T, Thavendiranathan P. Emerging Cardiac Imaging Modalities for the Early Detection of Cardiotoxicity Due to Anticancer Therapies. Rev Esp Cardiol (Engl Ed). 2017 Jun;70(6):487-495. English, Spanish. doi: 10.1016/j.rec.2017.01.004. Epub 2017 Feb 8. PMID: 28189542.

[5] López-Fernández, Teresa, and Paaladinesh Thavendiranathan. "Emerging Cardiac Imaging Modalities for the Early Detection of Cardiotoxicity Due to Anticancer Therapies." Revista 
Española De Cardiología (English Edition), vol. 70, no. 6, 2017, pp. 487-495., doi:10.1016/j.rec.2017.01.004.

[6] Cerqueira, M. D., Weissman, N. J., Dilsizian, V., Jacobs, A. K., Kaul, S., Laskey, W. K., ... Verani, M. S. (2002). Standardized myocardial segmentation and nomenclature for tomographic imaging of the heart. A statement for healthcare professionals from the Cardiac Imaging Committee of the Council on Clinical Cardiology of the American Heart Association. The international journal of cardiovascular imaging, 18(1), 539-542.

[7] Jung, Joonho, et al. "Patient-Specific 17-Segment Myocardial Modeling on a Bull's-Eye Map." Journal of Applied Clinical Medical Physics, vol. 17, no. 5, 2016, pp. 453-465., doi:10.1120/jacmp.v17i5.6237.

[8] Selvadurai, Breyoni S.N., et al. "Definition of Left Ventricular Segments for Cardiac Magnetic Resonance Imaging.” JACC: Cardiovascular Imaging, vol. 11, no. 6, 2018, pp. 926928., doi:10.1016/j.jcmg.2017.09.010.

[9] Berman DS, Abidov A, Kang X, Hayes SW, Friedman JD, Sciammarella MG, Cohen I, Gerlach J, Waechter PB, Germano G, Hachamovitch R. Prognostic validation of a 17-segment score derived from a 20-segment score for myocardial perfusion SPECT interpretation. J Nucl Cardiol. 2004 Jul-Aug;11(4):414-23. doi: 10.1016/j.nuclcard.2004.03.033. PMID: 15295410. 
[10] Vira T, Knudtson M, Merchant N, Howarth A. Application of the AHA 17 Segment Model: Evaluation of Relative Segmental Mass of the Left Ventricle by Cardiac MRI. Circulation. 2018 Mar 2018. Abstract.

[11] Andre, F., Lehrke, S., Katus, H.A. et al. Reference values for the left ventricular wall thickness in cardiac MRI in a modified AHA 17-segment model. J Cardiovasc Magn Reson 14, P223 (2012). https://doi.org/10.1186/1532-429X-14-S1-P223

[12] Talaat, Mohamed, et al. "The Coronary Arterial Anatomy of the 17-Segment Model Using 3-Tesla Cardiac Magnetic Resonance Imaging." The Egyptian Journal of Radiology and Nuclear Medicine, vol. 47, no. 4, 2016, pp. 1303-1311., doi:10.1016/j.ejrnm.2016.06.021.

[13] Lichtenauer M, Schreiber C, Jung C, Beer L, Mangold A, Gyöngyösi M, Podesser BK, Ankersmit HJ. Myocardial infarct size measurement using geometric angle calculation. Eur J Clin Invest. 2014 Feb;44(2):160-7. doi: 10.1111/eci.12202. Epub 2013 Dec 19. PMID: 25266895.

[14] Frankart, M., Arsanjani, R., Unzek, S., \& Yang, M. (2019). Application of the Left Ventricle 17-Segment Model in Delineation of Transthyretin Cardiac Amyloidosis Diagnosed on Myocardial Tc-99m Pyrophosphate Scintigraphy.

[15] Elisa McAlindon, Maria Pufulete, Chris Lawton, Gianni D. Angelini, Chiara BucciarelliDucci, Quantification of infarct size and myocardium at risk: evaluation of different techniques 
and its implications, European Heart Journal - Cardiovascular Imaging, Volume 16, Issue 7, July 2015, Pages 738-746, https://doi.org/10.1093/ehjci/jev001

[16] Rajiah P, Desai MY, Kwon D, Flamm SD. MR imaging of myocardial infarction. Radiographics. 2013 Sep-Oct;33(5):1383-412. doi: 10.1148/rg.335125722. PMID: 24025931. 


\section{Figures:}

\section{Figure 1.}

Fig. 1: Methods of Infarct Sizing. Different contouring techniques and standard infarct sizing measuring techniques displayed. (a) 17-segment model displaying different regions of the heart from apex (17) to base (1-6) used to measure SAX MRI on a standardized scale that associates each infarcted area with $\sim 5.88 \%$ infarct. (b) SAX infarct size contouring performed on a representative late gadolinium enhancement short-axis of the left ventricle. Contours are shown with the epicardium (light green), endocardium (red) and infarct (yellow) all traced. (c) Histopathology slice with infarct size contouring shown with the epicardium (light green), endocardium (red) and infarct (yellow) all traced. 


\section{Figure 2.}

Fig. 2: LAX infarct measurements (yellow) shown on 2-Chamber (a), 3-Chamber (b) and 4Chamber (c) planes. Total heart length (THL from apex to base (red) is taken in the 2-Chamber plane in order to standardize infarct size measuring across CMR (d). The 2 Chamber infarct measurement yields a value of 40.4 millimeters (a). The 3 Chamber infarct measurement yields a value of 90.8 millimeters (b). The 4 Chamber infarct measurement yields a value of 68.4 millimeters (c). The THL measurement yields a value of 91.7 millimeters (d). In this example, the summed multiplane LAX infarct length is 199.6, which is divided by the THL of $91.7 \mathrm{~mm}$, providing a Long-Axis Ratio (LAR) value of 2.18, which corresponds to an LV infarct percentage of $17.6-19.4 \%$ (Table 1$)$. 


\section{Figure 3.}

Fig. 3: Scatter plot comparing the LAR values to MRI contoured LV infarct percentages. The scatter plot (a) demonstrated a high correlation $\left(\mathrm{R}^{2}=0.911\right)$ between LAR values and LV Infarct percentages with corresponding data. Data were both taken from the terminal MRI so values are relatable. 


\section{Table 1.}

Table 1: LAR values can be used to give a corresponding LV infarct percentage. LAR values were plotted against MRI contoured infarct percentages in figure 3A. Since the graph displayed a high $\mathrm{R}^{2}$ value the linear trendline was used to create the above reference table to relate relative LAR values to an LV infarct percentage so that the observer can have a quantitative analysis providing both infarct length measurements along each wall of the heart and an LV infarct percentage. 
Table 2.

Table 2: Histopathology infarct percentage (from post-mortem heart analysis), 17-Segment model terminal MRI infarct percentage, MRI contour terminal MRI infarct percentage. Data from the cohort are averaged with standard error for each respective technique. Corresponding minimum and maximum data are shown in parenthesis. Paired T-Test values are shown against histopathology infarct percentage with no significant differences to be expected. 
Figure 4

Fig. 4: Bland-Altman Plot. Bland-Altman plot depicting mean difference for AHA 17-segment model infarct size versus histopathology (a) and MRI contoured infarct size versus histopathology infarct size (b) and limits of agreement.

b. 


\section{Figure 5.}

Fig. 5: CMR image of a patient with an anterior/apical MI. Clinical Applicability of LAR Infarct Sizing. Human MRI shows that LAR values can be contoured to match similar processing as in swine. The 2, 3, and 4 Chamber views of the infarct are contoured in yellow. The 4 Chamber infarct measurement yields a value of 9.5 centimeters (c). The THL measurement is shown in red and can be used to create a LAR and then reference Table 1 to find a relative infarct percentage. 
Figures
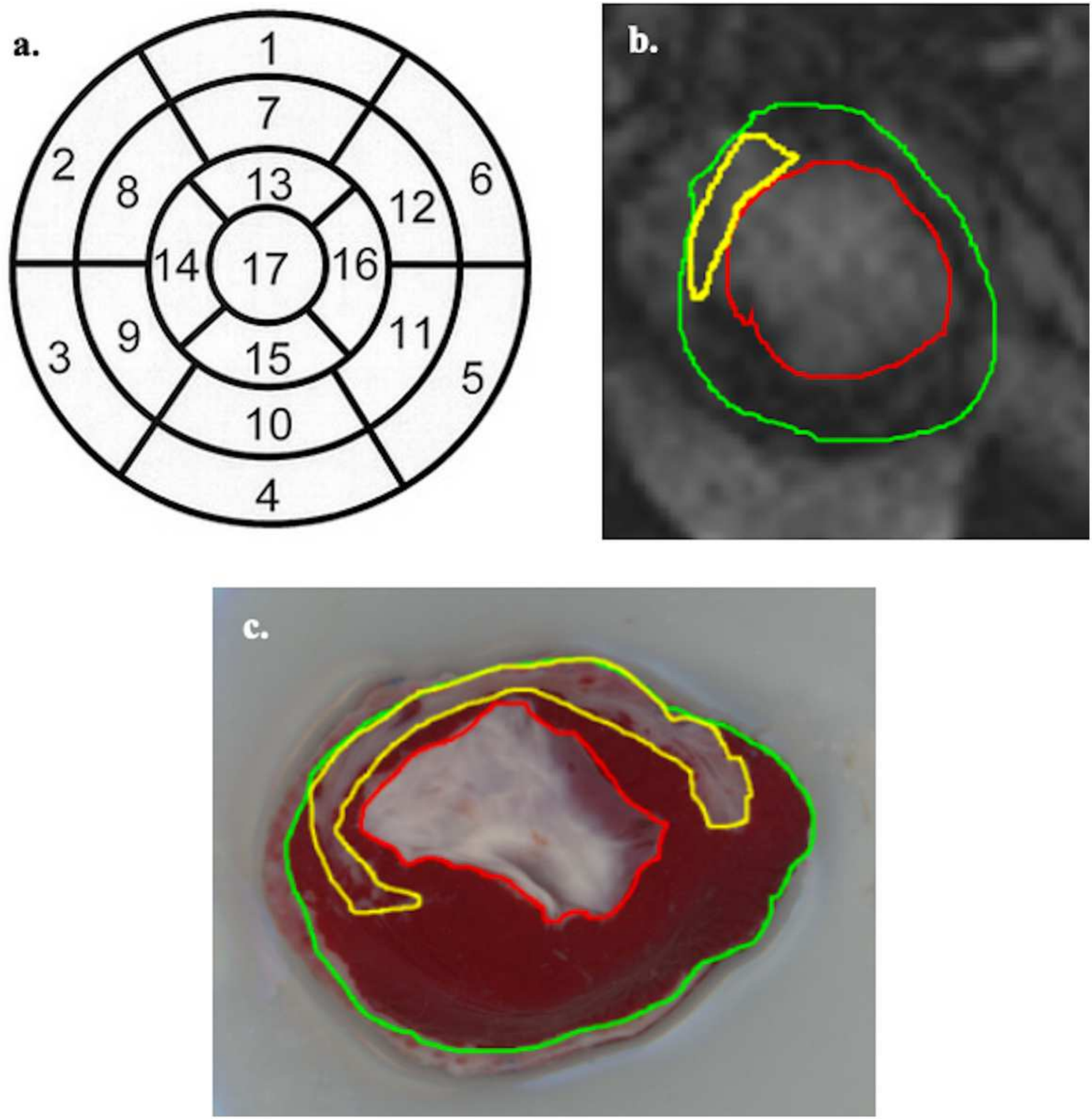

Figure 1

Methods of Infarct Sizing. Different contouring techniques and standard infarct sizing measuring techniques displayed. (a) 17-segment model displaying different regions of the heart from apex (17) to base (1-6) used to measure SAX MRI on a standardized scale that associates each infarcted area with $\sim 5.88 \%$ infarct. (b) SAX infarct size contouring performed on a representative late gadolinium 
enhancement short-axis of the left ventricle. Contours are shown with the epicardium (light green), endocardium (red) and infarct (yellow) all traced. (c) Histopathology slice with infarct size contouring shown with the epicardium (light green), endocardium (red) and infarct (yellow) all traced.
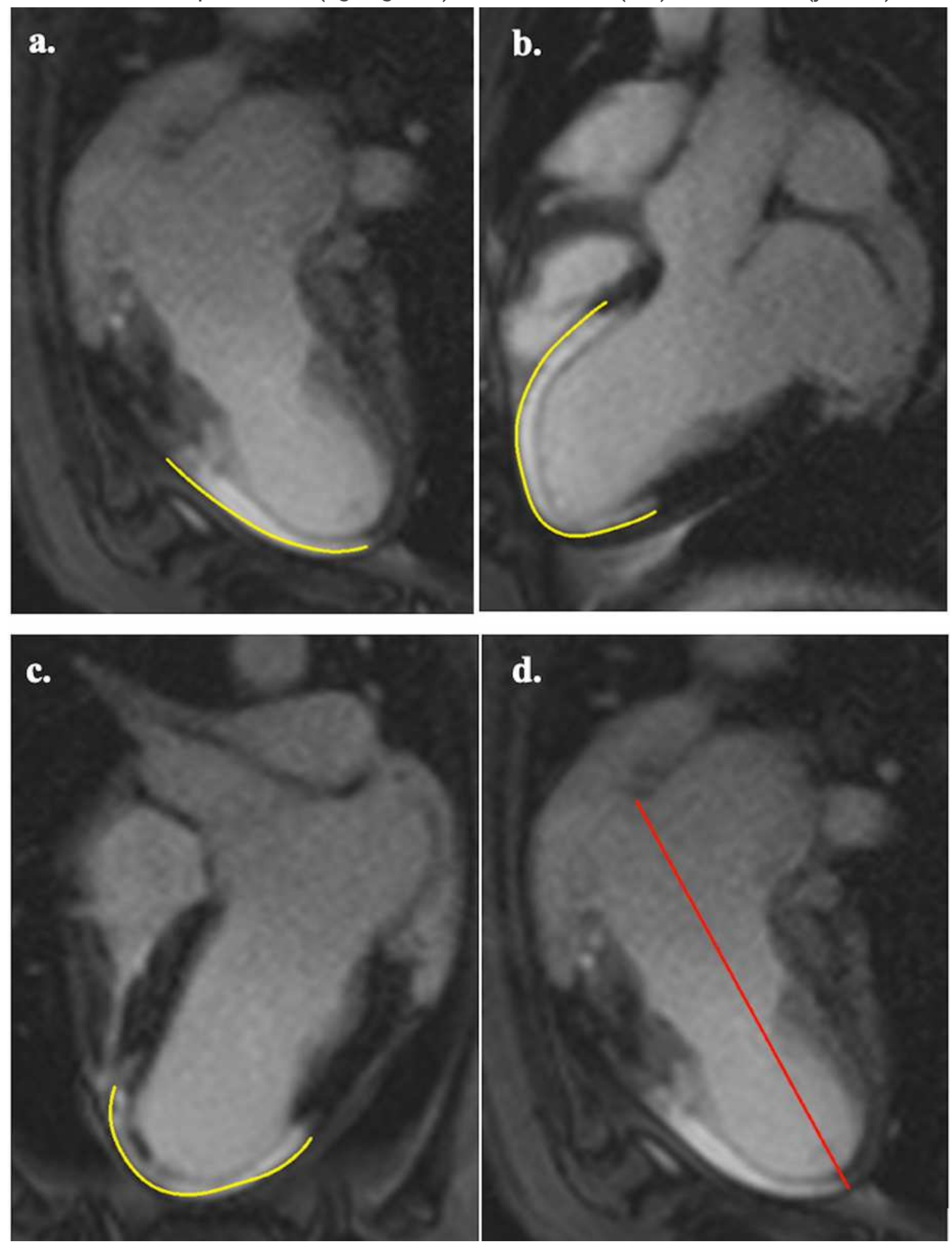

d.

\section{Figure 2}

LAX infarct measurements (yellow) shown on 2-Chamber (a), 3-Chamber (b) and 4-Chamber (c) planes. Total heart length (THL from apex to base (red) is taken in the 2-Chamber plane in order to standardize 
infarct size measuring across CMR (d). The 2 Chamber infarct measurement yields a value of 40.4 millimeters (a). The 3 Chamber infarct measurement yields a value of 90.8 millimeters (b). The 4 Chamber infarct measurement yields a value of 68.4 millimeters (c). The THL measurement yields a value of 91.7 millimeters (d). In this example, the summed multiplane LAX infarct length is 199.6, which is divided by the THL of $91.7 \mathrm{~mm}$, providing a Long-Axis Ratio (LAR) value of 2.18, which corresponds to an LV infarct percentage of $17.6-19.4 \%$ (Table 1).

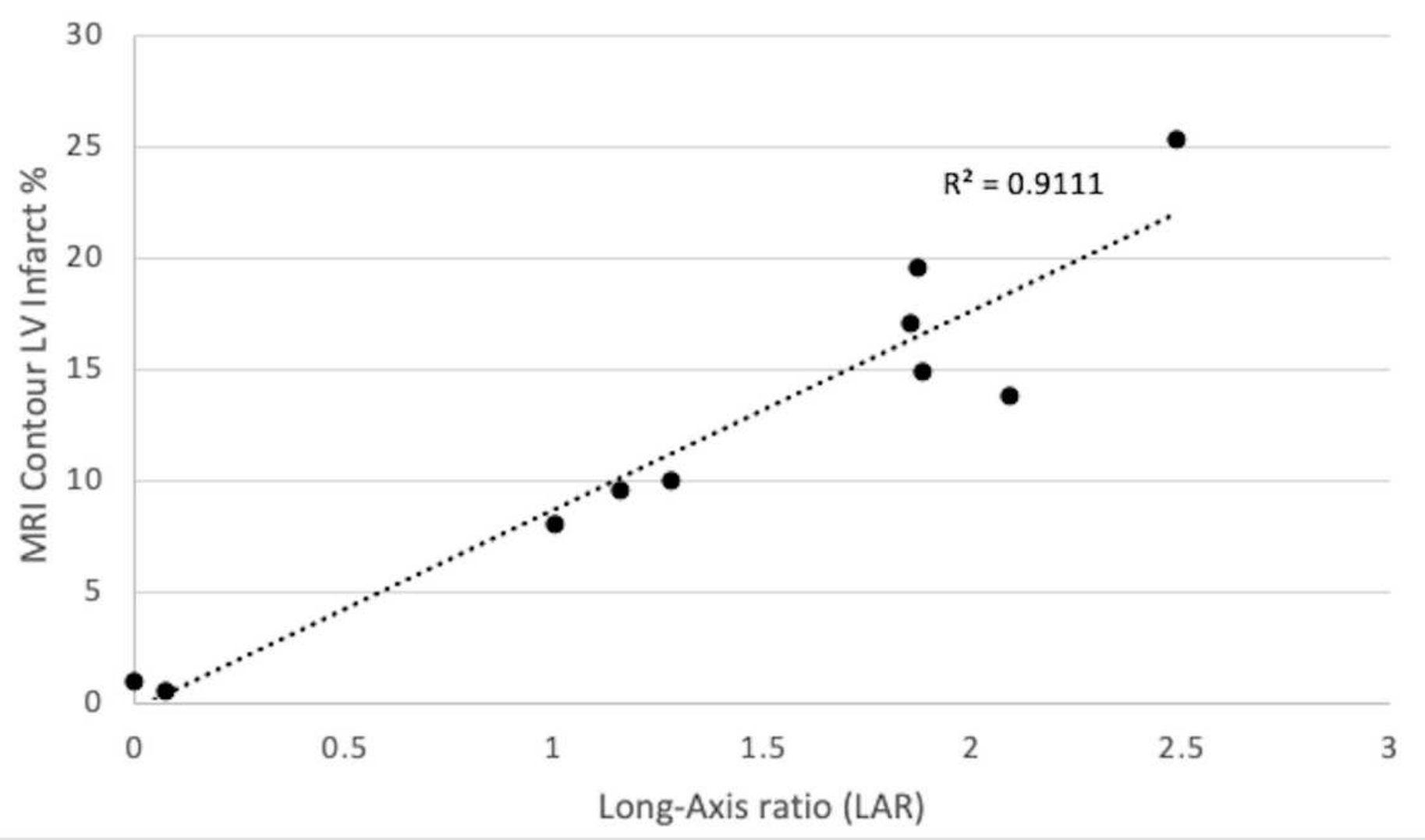

\section{Figure 3}

Scatter plot comparing the LAR values to MRI contoured LV infarct percentages. The scatter plot (a) demonstrated a high correlation $(R 2=0.911)$ between LAR values and LV Infarct percentages with corresponding data. Data were both taken from the terminal MRI so values are relatable. 
a.

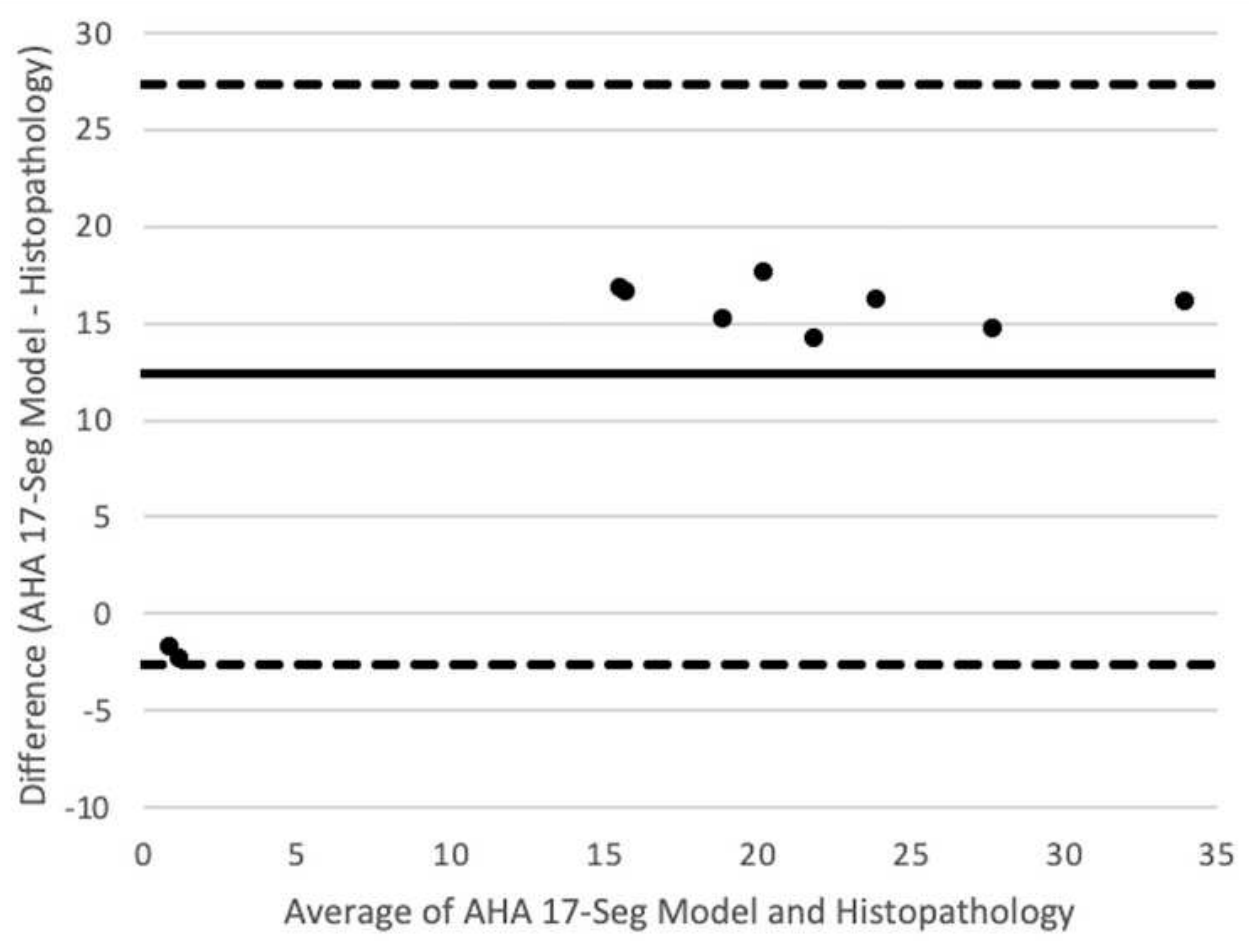

b.

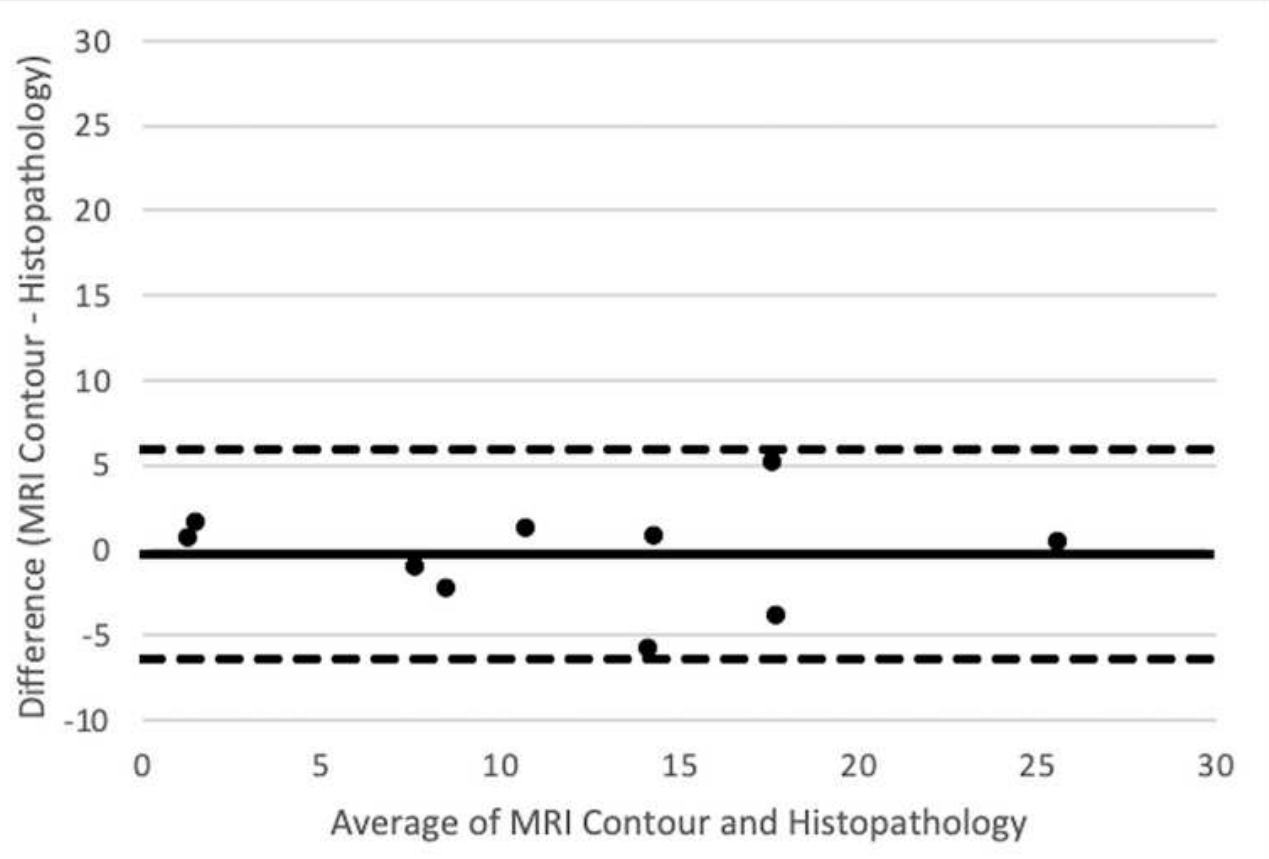

Figure 4

Bland-Altman Plot. Bland-Altman plot depicting mean difference for AHA 17-segment model infarct size versus histopathology (a) and MRI contoured infarct size versus histopathology infarct size (b) and limits of agreement. 

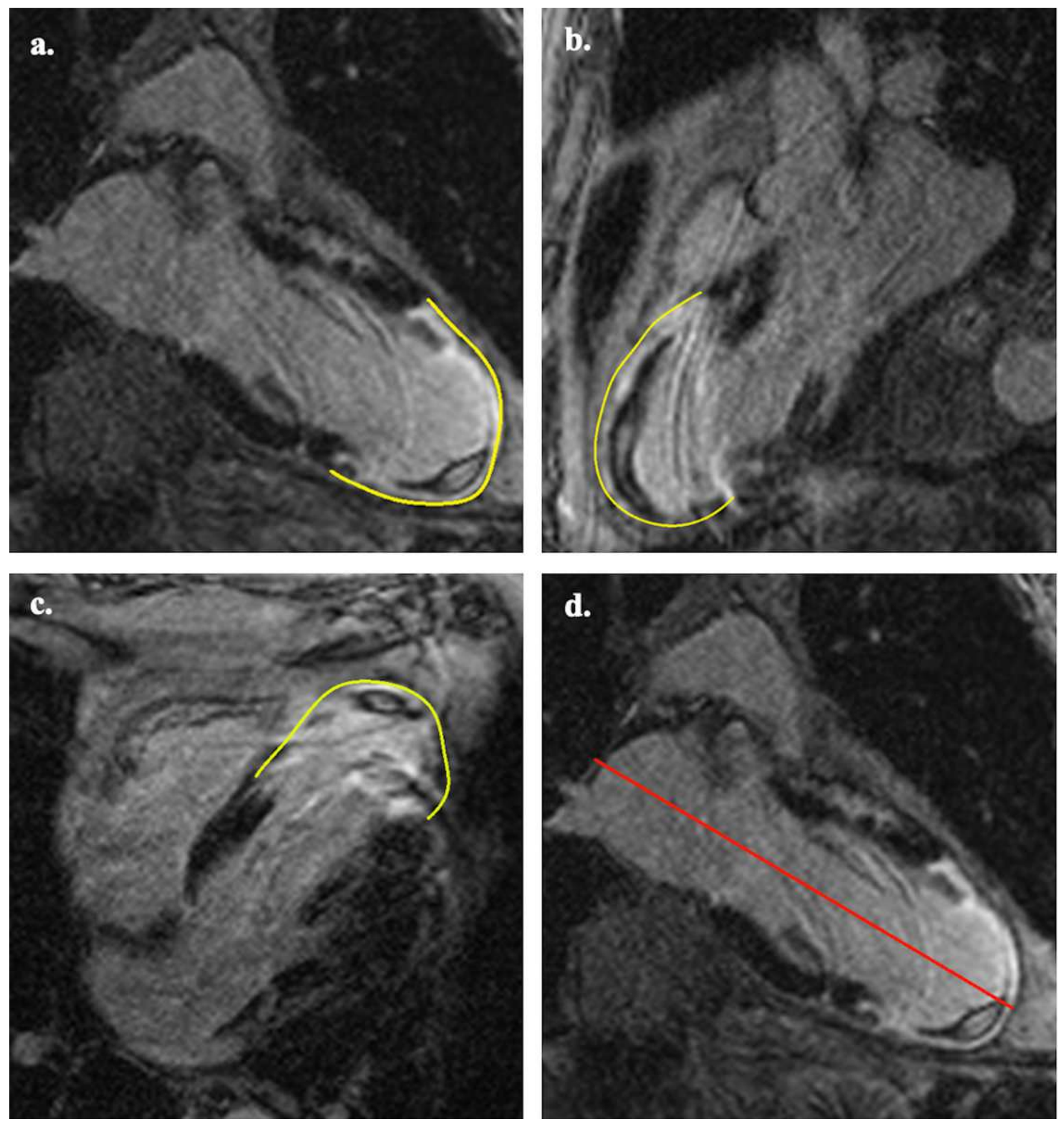

\section{Figure 5}

CMR image of a patient with an anterior/apical MI. Clinical Applicability of LAR Infarct Sizing. Human MRI shows that LAR values can be contoured to match similar processing as in swine. The 2,3 , and 4 Chamber views of the infarct are contoured in yellow. The 4 Chamber infarct measurement yields a value of 9.5 centimeters (c). The THL measurement is shown in red and can be used to create a LAR and then reference Table 1 to find a relative infarct percentage. 